\title{
Further assessment of deceleration-time histories for occupant injury and the damage of protected object in a crash stop
}

DOI:

10.1016/j.jijimpeng.2019.04.013

\section{Document Version}

Accepted author manuscript

Link to publication record in Manchester Research Explorer

Citation for published version (APA):

Yang, C., \& Li, Q. M. (2019). Further assessment of deceleration-time histories for occupant injury and the damage of protected object in a crash stop. International Journal of Impact Engineering, 130, 184-191.

https://doi.org/10.1016/j.jijimpeng.2019.04.013

\section{Published in:}

International Journal of Impact Engineering

\section{Citing this paper}

Please note that where the full-text provided on Manchester Research Explorer is the Author Accepted Manuscript or Proof version this may differ from the final Published version. If citing, it is advised that you check and use the publisher's definitive version.

\section{General rights}

Copyright and moral rights for the publications made accessible in the Research Explorer are retained by the authors and/or other copyright owners and it is a condition of accessing publications that users recognise and abide by the legal requirements associated with these rights.

\section{Takedown policy}

If you believe that this document breaches copyright please refer to the University of Manchester's Takedown Procedures [http://man.ac.uk/04Y6Bo] or contact uml.scholarlycommunications@manchester.ac.uk providing relevant details, so we can investigate your claim.

\section{OPEN ACCESS}


1 Further assessment of deceleration-time histories for occupant injury

\section{and the damage of protected object in a crash stop}

Chengxing Yang, Q. M. Li*

School of Mechanical, Aerospace and Civil Engineering, The University of Manchester, Pariser Building,

Manchester M13 9PL, UK

\begin{abstract}
Crash signal is generally given by a deceleration-time (or a force-time) history measured in a crash stop. Occupant injury and damage of protected object are highly related to the parameters of the crash signal. This paper assesses deceleration-time histories in a crash stop from two aspects, i.e., (i) minimisation of head injury index; (ii) physical modelling using single-degree-of-freedom (SDOF) model. A mathematical description for the determination of the safest deceleration-time history for head injury is presented, and seven well-recognised equivalent crash signals are analysed with constrains of identical velocity change and stop distance. Iso-damage/injury boundaries based on both methods are constructed using non-dimensional quantities. Results show that the preferred deceleration-time history depends on the selection of the injury (or damage) criterion and the ranges of parameters in the crash stop problem. These findings may support the design of cushioning or energy absorbing systems for the crashworthiness of vehicles, air and space crafts, ships and packages.
\end{abstract}

Keywords: Deceleration-time history; Crash stop; Head injury criteria (HIC); Single-degree-of-freedom (SDOF); Impact energy absorption (IEA)

*Corresponding author; e-mail address: qingming.li@manchester.ac.uk (Q.M. Li). 


\section{Introduction}

The safety of occupants and any other protected objects (e.g. goods, devices, equipment, etc.) is a critical issue in transportation, which promotes the continuous improvement of the crashworthiness safety of land, water (sea), air and space vehicles. The deceleration-time history in a crash incident has been commonly used as a crash signal for the assessment of the structural crashworthiness, occupant injury and the damage of protected objects. Deceleration-time histories may have different pulse shapes, time durations and peak values, as shown in [1] in rear impact tests. Numerical method has been adopted to evaluate the effect of crash pulse shape on occupant [2-4]. In Ref.[4], various shapes of crash signals (e.g. triangular , half-sine and square shapes) with identical velocity change $(\Delta V)$ were taken as inputs to a crash dummy model to determine how occupant reacts to different deceleration pulses where air bag and the knee bolster were applied as restraint systems in the simulation model. It showed that the time duration $\left(t_{d}\right)$, the peak deceleration $\left(A_{\max }\right)$, and the shape of the crash signal may influence the occupant response. When $t_{d}$ is given, the square signal was found to yield smaller peak acceleration of driver chest. Zhou et al. [5] analytically modelled the deceleration pulse of FMVSS201 head impact using an asymmetric haversine wave with the consideration of rebounding and found that the HIC is sensitive to impact velocity and stop distance $(\Delta S)$.

Strong correlation has been observed between deceleration and occupant injury by analysing the real-life crashes and dynamic tests [6]. In rear impacts, it was noted that the AIS-1 neck injury in rear impacts is relevant to both the mean deceleration $\left(\mathrm{A}_{\mathrm{m}}\right)$ and $\Delta V$ [7]. The safest crash signal shape has attracted attentions in the study of vehicle crash and restraint system [6,8-10]. Therefore, it is interesting to know the most preferred crash signal for the safety of occupants in order to develop more efficient crashworthy structure, impact energy absorber (IEA) [11-12], restraint system and shock absorber [13]. Effects of typical crash signals with identical $\Delta V$ and $\Delta S$ on head injury criteria (HIC) have been studied by Chou and Nyquist [14], Lim et al. [15,16], Huang [6], Wu and Beaudet [17] and Hutchinson et al. [18]. Wu and Beaudet [17] further proposed an ideal crash signal in the form of negative power function.

Crash stop is a common phenomenon in many natural and engineering activities, e.g., crash stop of transportation vehicles, accidental and deliberated crash-landing of containers, re-entry capsules and personal electronics, animal behaviours such as bird pecking, etc. During a crash stop, deceleration-time history $(A(t))$ of the concerned occupant, determined by the energy absorbing mechanism of the surrounding supporting structure and IEA, is usually used to assess the occupant injury or damage. The design of an IEA is normally required to bring a safe stop of the occupant for the given maximum survivable impact speed (i.e. the velocity change) and the 
available stop distance. In many application fields, the stop distance is referred to the cushioning space $(\Delta S)$, which is usually limited by the product or system space available for cushioning purpose. For example, the collapse zone of rail vehicles used to absorb impact energy in crash accidents is mounted on the front-end of the underframe. This zone is restricted by other vehicle components, e.g., survival space, support beams, couplers, etc. Therefore, it is a generic problem to stop a moving object under the constraints of velocity change and stop distance.

The objective of the present work is to further assess the deceleration-time history $(A(t))$ for given stop distance $(\Delta S)$ and velocity change $(\Delta V)$ in a crash stop incident. Two different well-known methods, i.e. empirical HIC and equivalent single degree of freedom (SDOF) model, are employed. Without causing confusion and for the simplification, the term of 'occupant' is used for human body/part and the term of 'protected object', although it may include occupant, is mainly used for any other goods, products and equipment to be protected; the term of 'injury' is related to an occupant while the term of 'damage' is associated with a protected object; the term of 'deceleration' may include the acceleration and the term of 'crash signal' represents the deceleration-time (or force-time) history, if no particular notation is given.

\section{Determination of deceleration-time history based on HIC}

The injury or damage of a protected object is a major concern in a crash stop. A crashworthy system can be generally divided into two parts, i.e., the cushioning component (e.g. damping system, energy-absorbing structures, shock absorbers, etc.) and the protected object (e.g. human, electronic components and devices, nuclear waste containers, goods, equipment). Fig.1(a) illustrates a generic crash model, which is generally simplified into the cushioning zone consisting of cushioning components (IEA and restrains) and the concerned envelope of the protected object. Without losing generality, the protected object could be understood as either the envelope of the rigid survival space of a vehicle or the envelope of a human body (or part) depending on the statement of the problem. For a packaging problem, the cushioning in Fig.1(a) represents the package and the protected object is the product inside the package. The deceleration-time history $(A(t))$ applied to the protected object is determined by the design of cushioning zone while the safety of the protected object (or occupant) can be assessed by the associated damage (or injury) criteria in terms of $A(t)$ shown in Fig.1(b) as a typical crash signal acting on the protected object. If the damage/injury index can be expressed as a function of $A(t)$, i.e., $F(A(t))$ where $A(t)=0$ when $t \leq 0$ and $t \geq t_{d}$ with $t_{d}$ as the duration of the crash time, then the safest deceleration-time history $A_{s}(t)$ for a given stop distance $(\Delta S)$ and initial impact speed $\left(V_{0}\right)$ is determined by

$$
F\left(A_{s}(t)\right)=\min F(A(t)) \|\left(V_{0}-\int_{0}^{t_{d}} A(t) d t=0, \int_{0}^{t_{d}}\left[V_{0}-\int_{0}^{t} A(t) d t\right] d t=S\right)
$$


Here, the residual velocity is assumed to be zero without rebounding, which means $V_{0}=\Delta V$. Mathematically, the safest deceleration-time history $A_{s}(t)$ is determined by the minimum of $F(A(t))$ in Eq.(1).

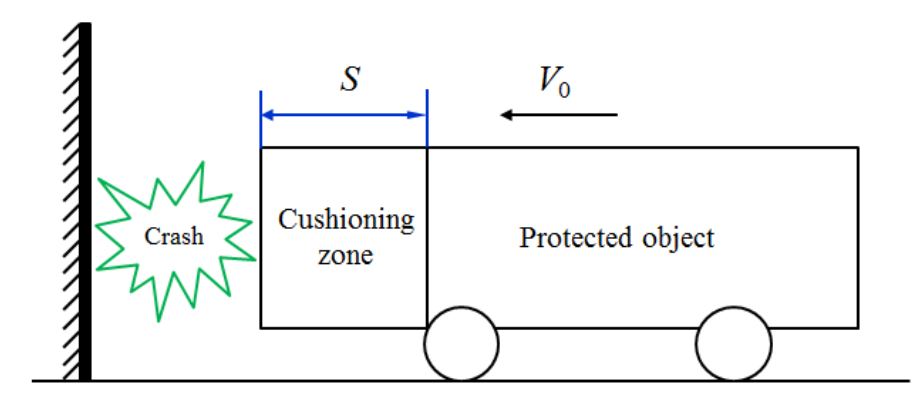

(a)

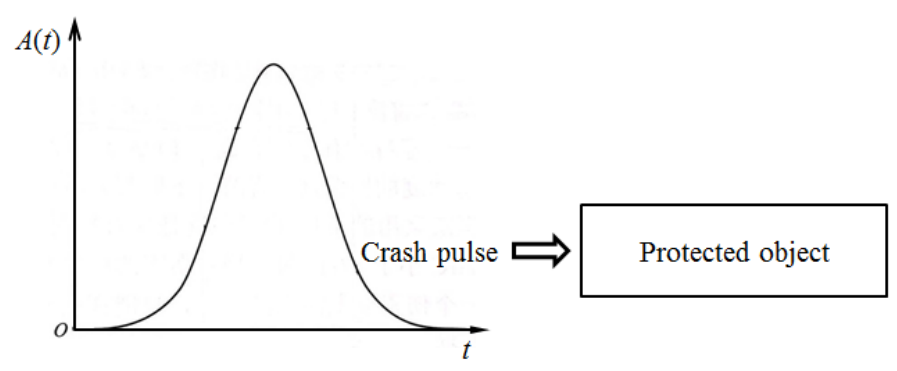

(b)

Fig.1. (a) A generic crash senario; (b) A typical crash pulse acting on the protected object.

In a frontal vehicle crash, the deceleration $A(t)$ at the centroid of an occupant's head is a function of time $(t)$. HIC was firstly proposed by the National Highway Traffic Safety Administration (NHTSA) in 1972. This criterion is based on the average value of the deceleration over the most critical part of $A(t)$, which is calculated using the formula below

$H I C=\max _{t_{1}, t_{2}}\left[\left(t_{2}-t_{1}\right) \cdot\left(\frac{1}{t_{2}-t_{1}} \int_{t_{1}}^{t_{2}} A(t) d t\right)^{2.5}\right]=\left(t_{2}^{*}-t_{1}^{*}\right) \cdot\left(\frac{1}{t_{2}^{*}-t_{1}^{*}} \int_{t_{1}^{*}}^{t_{2}^{*}} A(t) d t\right)^{2.5}$

where $t_{1}$ and $t_{2}$ satisfy $0 \leq t_{1}<t_{2} \leq t_{d}$ and the maximum value of the HIC function in Eq.(2) is achieved when $t_{1}=t_{1}^{*}$ and $t_{2}=t_{2}^{*}$; deceleration $A(t)$ and time $t$ are measured in standard gravity acceleration $(g ' s)$ and second, respectively. Normally, the interval $\Delta t=t_{2}^{*}-t_{1}^{*}$ is $36 \mathrm{~ms}$ [19] or $15 \mathrm{~ms}$ [20].

\subsection{Determination of $A(t)$ by HIC formula}

To elucidate the preferred $A(t)$ in a crash stop, effects of seven typical and well-recognised equivalent crash signals (ECSs) on HIC are analysed, which are equivalent square signal (ESS), equivalent positive triangular signal (EPTS), equivalent negative triangular signal (ENTS), equivalent isosceles triangular signal (EITS), equivalent half sine signal (EHSS), equivalent haversine signal (EHVS) and the equivalent negative power signal (ENPS) proposed by Wu and Beaudet [17]. It is noted that the term of 'equivalent crash wave' used in some publications 
111 (e.g.[6]) has the same meaning of ECS. To compare their injury indices in a crash stop incident, all 112 seven considered ECSs shown in Fig.2 will have identical $\Delta V$ and $\Delta S$.

113

114

115

116

117

118

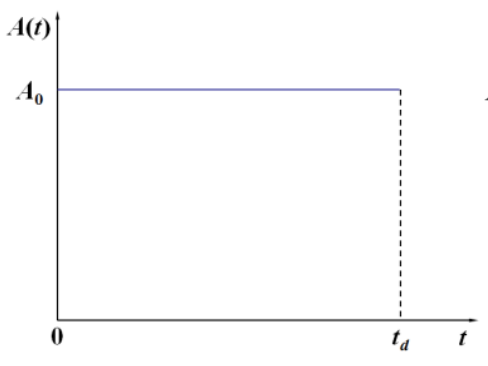

(a)

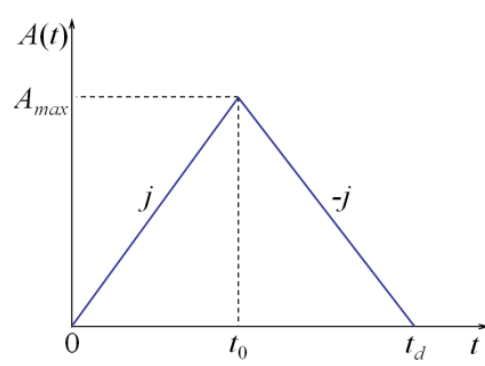

(d)

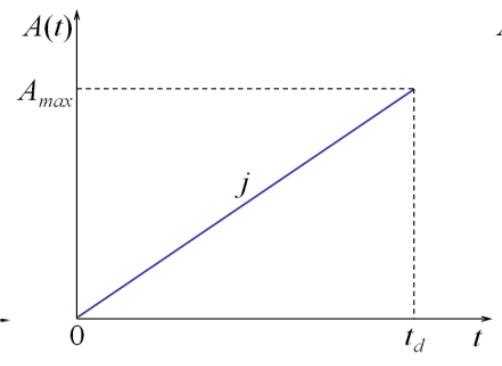

(b)

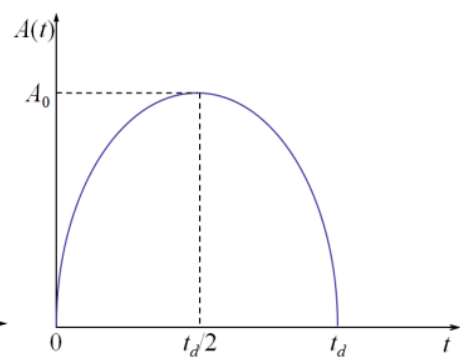

$(e)$

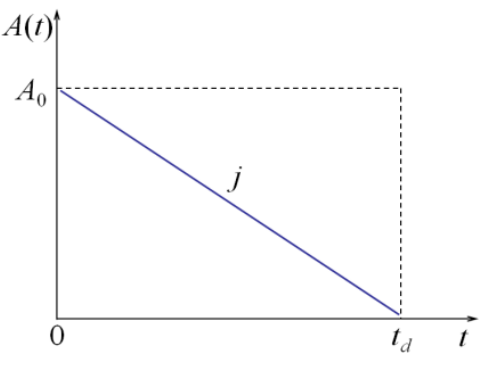

(c)

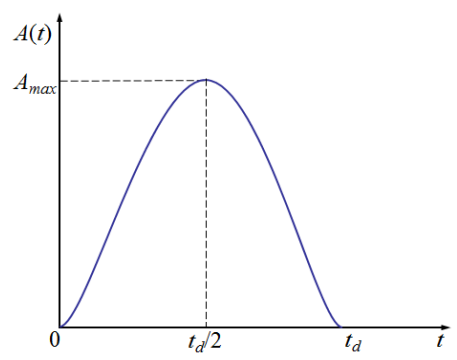

(f)

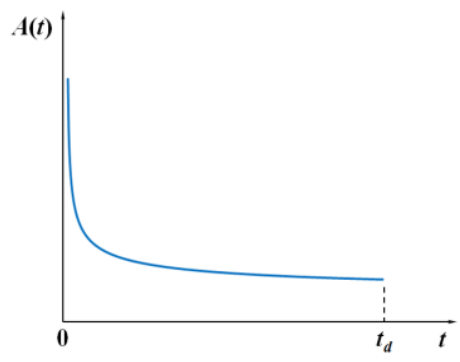

( $g$ )

Fig.2. Typical shapes of ECSs: (a) ESS; (b) EPTS; (c) ENTS; (d) EITS; (e) EHSS; (f) EHVS; (g) ENPS.

The time duration $t_{d}$ and maximum deceleration $A_{\max }$ may vary among the seven signals in Fig.2 for given $\Delta V$ and $\Delta S$. The results are tabulated in Table 1, which include $t_{1}^{*}$ and $t_{2}^{*}$ in HIC calculations, expressions of crash signals and expressions of HIC. The normalised HIC value against each ECS is also shown in Fig.3. Details of the calculation processes are referred to Chou and Nyquist [14], Lim et al. [15], Huang [6] and Wu and Beaudet [17].

Table 1. HIC derived from ECSs with identical $\Delta V$ and $\Delta S$. (Units of $A_{\max }, t_{d}, \Delta V$ and $\Delta S$ are $9.8 \mathrm{~m} / \mathrm{s}^{2}, \sec , 9.8 \mathrm{~m} / \mathrm{s}$ and $9.8 \mathrm{~m}$, respectively)

\begin{tabular}{c|c|c|c|c|c|c|c}
\hline Crash signals & ESS & EPTS & ENTS & EITS & EHSS & EHVS \\
\hline Expression of signal & $A_{0}$ & $j t$ & $A_{0}-j t$ & $j t\left(0 \leq t \leq t_{0}\right)$ & ENPS & $A_{0} \sin \omega t$ & $\frac{A_{\max }}{2}[1-\cos \omega t]$ \\
\hline
\end{tabular}




\begin{tabular}{|c|c|c|c|c|c|c|c|}
\hline $\begin{array}{c}\text { Times for the } \\
\text { maximum value of } \\
\text { HIC function }\end{array}$ & $\left\{\begin{array}{l}t_{1}^{*}=0 \\
t_{2}^{*}=t_{d}\end{array}\right.$ & $\left\{\begin{array}{l}t_{1}^{*}=\frac{3}{7} t_{d} \\
t_{2}^{*}=t_{d}\end{array}\right.$ & $\left\{\begin{array}{l}t_{1}^{*}=0 \\
t_{2}^{*}=\frac{4}{7} t_{d}\end{array}\right.$ & $\left\{\begin{array}{l}t_{1}^{*}=\frac{3}{14} t_{d} \\
t_{2}^{*}=\frac{11}{14} t_{d}\end{array}\right.$ & $\left\{\begin{array}{l}t_{1}^{*}=\frac{0.518}{\omega} \\
t_{2}^{*}=\frac{2.624}{\omega}\end{array}\right.$ & $\left\{\begin{array}{l}t_{1}^{*}=0.25 t_{d} \\
t_{2}^{*}=0.75 t_{d}\end{array}\right.$ & $\left\{\begin{array}{l}t_{1}^{*}=0 \\
t_{2}^{*}=t_{d}\end{array}\right.$ \\
\hline$\Delta t^{*}=t_{2}^{*}-t_{1}^{*}$ & $\frac{2 \Delta S}{\Delta V}$ & $\frac{6 \Delta S}{7 \Delta V}$ & $\frac{12 \Delta S}{7 \Delta V}$ & $\frac{8 \Delta S}{7 \Delta V}$ & $\frac{4.212 \Delta S}{\pi \Delta V}$ & $\frac{\Delta S}{\Delta V}$ & $\frac{8 \Delta S}{3 \Delta V}$ \\
\hline Expression of HIC & $A_{0}^{2.5} t_{d}$ & $\frac{4}{7}\left(\frac{5}{7} j\right)^{2.5} t_{d}^{3.5}$ & $\frac{4}{7 j}\left(\frac{5}{7}\right)^{2.5}\left(A_{0}\right)^{3.5}$ & $\left(\frac{7}{8}\right)^{1.5}\left(\frac{40}{49} j\right)^{2.5}\left(\frac{t_{d}}{2}\right)^{3.5}$ & $1.303 \frac{\left(A_{0}\right)^{2.5}}{\omega}$ & $0.303\left(A_{\max }\right)^{2.5} t_{d}$ & $3.586 c^{2.5}$ \\
\hline Configurations & $\left\{\begin{array}{l}A_{0}=\frac{(\Delta V)^{2}}{2 \Delta S} \\
t_{d}=\frac{2 \Delta S}{\Delta V}\end{array}\right.$ & $\left\{\begin{array}{l}j=\frac{8(\Delta V)^{3}}{9(\Delta S)^{2}} \\
t_{d}=\frac{3 \Delta S}{2 \Delta V}\end{array}\right.$ & $\left\{\begin{array}{l}A_{0}=\frac{2(\Delta V)^{2}}{3 \Delta S} \\
j=\frac{2(\Delta V)^{3}}{9(\Delta S)^{2}} \\
t_{d}=\frac{3 \Delta S}{\Delta V}\end{array}\right.$ & $\left\{\begin{array}{l}A_{0}=\frac{2(\Delta V)^{2}}{\Delta S} \\
j=\frac{(\Delta V)^{3}}{(\Delta S)^{2}} \\
t_{d}=\frac{2 \Delta S}{\Delta V} \\
t_{0}=\frac{\Delta S}{\Delta V}\end{array}\right.$ & $\left\{\begin{array}{l}A_{0}=\frac{\pi(\Delta V)^{2}}{4 \Delta S} \\
\omega=\frac{\pi \Delta V}{2 \Delta S} \\
t_{d}=\frac{2 \Delta S}{\Delta V}\end{array}\right.$ & $\left\{\begin{array}{l}A_{\max }=0.9966 \frac{(\Delta V)^{2}}{\Delta S} \\
\omega=\frac{\pi \Delta V}{\Delta S} \\
t_{d}=\frac{2 \Delta S}{\Delta V}\end{array}\right.$ & $\left\{\begin{array}{l}c=\frac{3}{5}\left(\frac{8}{3} \Delta S\right)^{-0.6} \Delta V^{1.6} \\
t_{d}=\frac{8 \Delta S}{3 \Delta V}\end{array}\right.$ \\
\hline$A_{\max }$ & $\frac{(\Delta V)^{2}}{2 \Delta S}$ & $\frac{4}{3} \frac{(\Delta V)^{2}}{\Delta S}$ & $\frac{2}{3} \frac{(\Delta V)^{2}}{\Delta S}$ & $\frac{(\Delta V)^{2}}{\Delta S}$ & $\frac{\pi(\Delta V)^{2}}{4 \Delta S}$ & $0.9966 \frac{(\Delta V)^{2}}{\Delta S}$ & $+\infty$ \\
\hline$A_{\text {avg }}$ & $\frac{1}{2} \frac{(\Delta V)^{2}}{\Delta S}$ & $\frac{2}{3} \frac{(\Delta V)^{2}}{\Delta S}$ & $\frac{1}{3} \frac{(\Delta V)^{2}}{\Delta S}$ & $\frac{1}{2} \frac{(\Delta V)^{2}}{\Delta S}$ & $\frac{1}{2} \frac{(\Delta V)^{2}}{\Delta S}$ & $\frac{1}{2} \frac{(\Delta V)^{2}}{\Delta S}$ & $\frac{3}{8} \frac{(\Delta V)^{2}}{\Delta S}$ \\
\hline $\begin{array}{l}\text { Expression of HIC in } \\
\text { terms of } \Delta V \text { and } \Delta S\end{array}$ & $\begin{array}{l}(\Delta V)^{4} \\
\cdot(2 \Delta S)^{-1.5}\end{array}$ & $\begin{array}{l}2.15 \cdot(\Delta V)^{4} \\
\cdot(2 \Delta S)^{-1.5}\end{array}$ & $\begin{array}{l}0.76 \cdot(\Delta V)^{4} \\
\cdot(2 \Delta S)^{-1.5}\end{array}$ & $\begin{array}{l}1.39 \cdot(\Delta V)^{4} \\
\cdot(2 \Delta S)^{-1.5}\end{array}$ & $\begin{array}{l}1.28 \cdot(\Delta V)^{4} \\
\cdot(2 \Delta S)^{-1.5}\end{array}$ & $\begin{array}{l}1.7082 \cdot(\Delta V)^{4} \\
\cdot(2 \Delta S)^{-1.5}\end{array}$ & $\begin{array}{l}0.649502 \cdot(\Delta V)^{4} \\
\cdot(2 \Delta S)^{-1.5}\end{array}$ \\
\hline $\begin{array}{l}\text { Expression of HIC in } \\
\text { terms of } \Delta V \text { and } A_{\max }\end{array}$ & $\begin{array}{l}(\Delta V) \\
\cdot\left(A_{\max }\right)^{1.5}\end{array}$ & $\begin{array}{l}0.491(\Delta V) \\
\cdot\left(A_{\max }\right)^{1.5}\end{array}$ & $\begin{array}{l}0.491(\Delta V) \\
\cdot\left(A_{\max }\right)^{1.5}\end{array}$ & $\begin{array}{l}0.491(\Delta V) \\
\cdot\left(A_{\max }\right)^{1.5}\end{array}$ & $\begin{array}{l}0.649(\Delta V) \\
\cdot\left(A_{\max }\right)^{1.5}\end{array}$ & $0.604(\Delta V) \cdot\left(A_{\max }\right)^{1.5}$ & - \\
\hline $\begin{array}{l}\text { Expression of HIC in } \\
\text { terms of } \Delta V \text { and } A_{\text {avg }}\end{array}$ & $\begin{array}{l}(\Delta V) \\
\cdot\left(A_{\text {avg }}\right)^{1.5}\end{array}$ & $\begin{array}{l}1.39647(\Delta V) \\
\cdot\left(A_{\text {avg }}\right)^{1.5}\end{array}$ & $\begin{array}{l}1.39621(\Delta V) \\
\cdot\left(A_{\text {avg }}\right)^{1.5}\end{array}$ & $1.39(\Delta V) \cdot\left(A_{\text {avg }}\right)^{1.5}$ & $\begin{array}{l}1.28(\Delta V) \\
\cdot\left(A_{\text {avg }}\right)^{1.5}\end{array}$ & $1.7082(\Delta V) \cdot\left(A_{\text {avg }}\right)^{1.5}$ & $(\Delta V) \cdot\left(A_{\text {avg }}\right)^{1.5}$ \\
\hline
\end{tabular}

127 It is shown that $t_{1}^{*}$ and $t_{2}^{*}$ for the calculation of HIC are dependent of the specified ECS.

128 The interval $\left(\left[t_{1}^{*}, t_{2}^{*}\right]\right)$ of ENPS is the largest one, which equals the whole time duration $\left(\frac{8 S}{3 \Delta V}\right)$.

129 Interestingly, the expressions of $t_{2}^{*}-t_{1}^{*}$ derived from triangular signals are identical to $\frac{4}{7} t_{d}$, no

130 matter the signal is EPTS, ENTS or EITS. In other word, the HIC derived from all triangular

131 signals will be the same if $t_{d}$ (rather than the stop distance $\Delta S$ ) and $\Delta V$ are fixed. However, when

$132 \Delta V$ and $\Delta S$ are fixed, ENTS has the longest time duration of $t_{d}$ which is 1.5 times that of ESS,

133 while ENPS has the highest peak deceleration which approaches infinite.

134 It is found that the order of HIC values is ENPS $<E N T S<E S S<E H S S<E I T S<E H V S<E P T S$ for 135 given $\Delta V$ and $\Delta S$, as shown in Fig.3. Taking the ESS as a standard signal and its $\mathrm{HIC}=(\Delta V)^{4}$.

$136(2 S)^{-1.5}$ as a reference value, the normalised HIC values for ENPS, ENTS, ESS, EHSS, EITS, 137 EHVS and EPTS are 0.65, 0.76, 1, 1.28, 1.39, 1.84 and 2.15, respectively. It reveals that the ENPS 138 with high initial deceleration and longer time duration is preferable in reducing head injury, while 139 EPTS with gradually increasing deceleration and short time duration is the worst choice. These 140 observations are consistent with previous findings [14-17]. Furthermore, the orders of normalised 


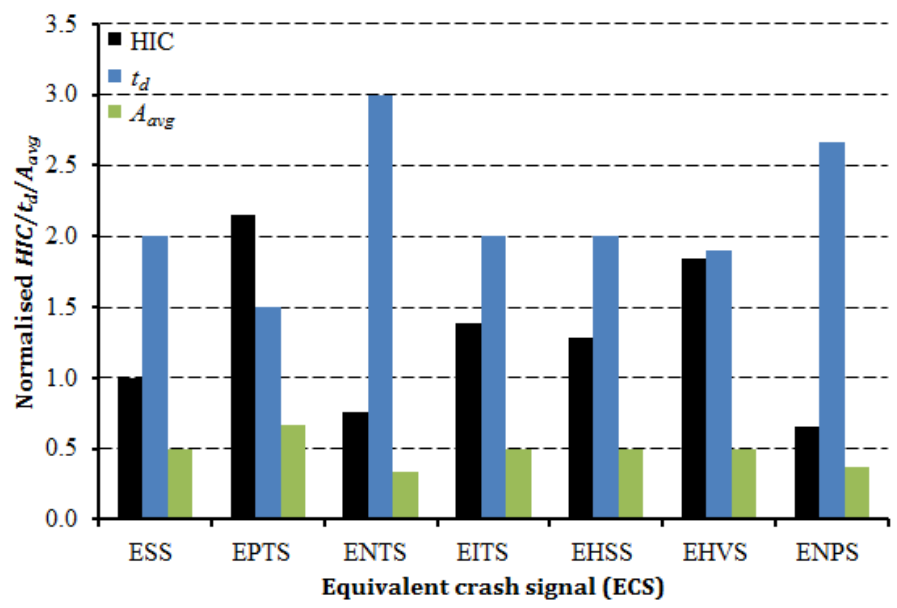

Fig.3. The normalised $H I C, t_{d}$ and $A_{\text {avg }}$ versus ECSs with identical $\Delta V$ and $\Delta S$.

\subsection{Determination of $A(t)$ by HIC-based injury boundary}

As discussed previously, $\Delta V$ and $\Delta S$ are the two critical parameters of the crash signal to affect HIC value. Therefore, it is convenient to use an iso-injury diagram on $\Delta S$ - $\Delta \mathrm{V}$ plane for a given critical value of $\mathrm{HIC}_{\mathrm{cr}}$ (e.g. $\mathrm{HIC}_{36}=\mathrm{HIC}_{\mathrm{cr}}=1000$ [21]). However, it should be noted that different values of $\mathrm{HIC}_{\mathrm{cr}}$ could be given for different levels of Abbreviated Injury Scale (AIS) [7], which is applicable to following discussion on iso-injury (iso-damage) diagrams. The HIC-based $\Delta S-\Delta \mathrm{V}$ iso-injury diagram can be expressed as

$\Delta S=\alpha \frac{(\Delta V)^{\frac{8}{3}}}{\left(H I C_{c r}\right)^{\frac{2}{3}}}$

according to Table 1 where $\alpha$ is a constant depending on the shape of ECS (Table 2).

Similar to pressure-impulse (P-I) iso-damage diagram [22], the iso-injury diagram of A(t) can also be expressed on $A_{\max }-\Delta \mathrm{V}$ plane. Because the peak acceleration of ENPS is infinite, $A_{a v g}-\Delta \mathrm{V}$ iso-injury diagram is adopted here, which, however, does not prevent the use of $A_{\max }-\Delta \mathrm{V}$ iso-injury for ECSs with finite peak values. The HIC-based $A_{a v g}-\Delta \mathrm{V}$ iso-injury diagram can be expressed as

$A_{\text {avg }}=\frac{\Delta V}{t_{d}}=\beta\left(\frac{H I C_{c r}}{\Delta V}\right)^{\frac{2}{3}}$

according to Table 1 where $\beta$ is a constant depending on the shape of ECS (Table 2).

Table 2. Coefficients ( $\alpha$ and $\beta$ ) for different ECSs. 


\begin{tabular}{c|c|c|c|c|c|c|c}
\hline Coefficients & ESS & EPTS & ENTS & EITS & EHSS & EHVS & ENPS \\
\hline$\alpha$ & 0.5000 & 0.8329 & 0.4164 & 0.6228 & 0.5895 & 0.7145 & 0.3750 \\
\hline$\beta$ & 1.0000 & 0.8004 & 0.8006 & 0.8029 & 0.8483 & 0.6998 & 1.000 \\
\hline
\end{tabular}

The iso-injury diagrams derived for various ECSs are presented on $\Delta S$ - $\Delta \mathrm{V}$ plane (Fig.4(a)) and $A_{\text {avg }}-\Delta \mathrm{V}$ plane (Fig.4(b)). In Fig.4(a), the injury area is below the iso-injury diagram while it is above the iso-injury diagram in Fig.4(b). Both iso-damage diagrams in Fig.4 show that ENPS results in the safest iso-injury diagram among those seven ECSs, which is consistent with the results presented in $\mathrm{Wu}$ and Beaudet [17] although the sequences of iso-injury diagrams in Fig.4(a) and Fig.4(b) for different ECSs are different. It should be noted that the iso-injury diagrams in Fig.4(b) derived from ENPS is the same as that of ESS. Iso-injury diagrams calculated from all triangular ECSs are also identical no matter the ECS is EPTS, ENTS or EITS, which can be seen clearly from Table 1 . This is due to the fact that the $A_{a v g}-\Delta V$ iso-injury diagram is determined without the control of same stop distance $\Delta S$.

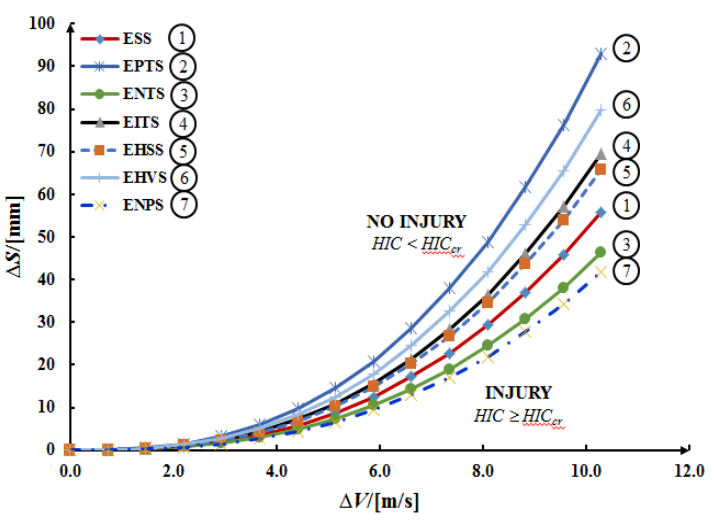

(a)

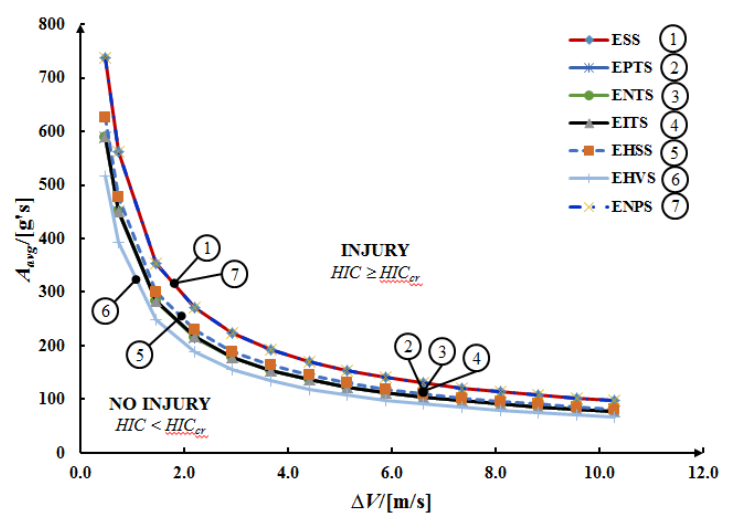

(b)

Fig.4. The iso-injury diagrams $\left(H I C_{c r}=1000\right)$ expressed on $(a) \Delta S-\Delta \mathrm{V}$ plane; (b) $A_{\text {avg }}-\Delta \mathrm{V}$ plane.

\section{Determination of $A(t)$ based on SDOF analysis}

SDOF model has been successfully used to obtain iso-damage diagrams for general structures subjected to blast loading [22-25], as well as impact loading such as package design [26-29]. Therefore, it is a useful tool for the damage assessment of a protected object subjected to deceleration during a crash stop. The SDOF is described by equivalent lump mass $\left(m_{b}\right)$, elastic stiffness $(k)$ and damping coefficient $(c)$ and is subjected to acceleration $\ddot{x}_{o}(t)=-A(t)$ at its base where $\mathrm{A}(\mathrm{t})$ is the deceleration-time history, as shown in Fig.5. Therefore, a SDOF model can represent a structural response inside the protected object when $A(t)$ determined by the IEA and cushion design is applied to the protected object. The dynamic response of SDOF can be described by

$m_{b} \ddot{x}_{b}+c\left(\dot{x}_{b}-\dot{x}_{o}\right)+k z_{b o}=0$ 
where $\left(z_{b o}=x_{b}-x_{o}\right)$ is the relative displacement and $\ddot{x}_{b}$ represents the absolute output acceleration.

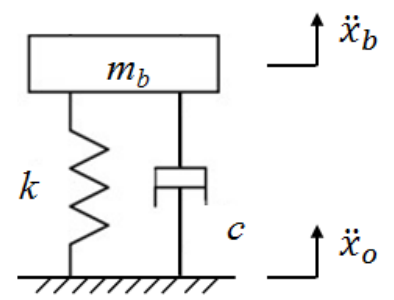

Fig.5. An SDOF model subjected to deceleration in a crash stop event.

Without losing the generality, damping effect is neglected, and the solutions of $\ddot{x}_{b}$ and $z_{b o}$ can be obtained by Duhamel integral, i.e.

$\ddot{x}_{b}(t)=\omega_{n} \int_{0}^{t} \ddot{x}_{o}\left(t^{\prime}\right) \sin \omega_{n}\left(t-t^{\prime}\right) d t^{\prime}$

$z_{b o}(t)=-\frac{1}{\omega_{n}} \int_{0}^{t} \ddot{x}_{o}\left(t^{\prime}\right) \sin \omega_{n}\left(t-t^{\prime}\right) d t^{\prime}$

where $t^{\prime}$ is middle variable and $\omega_{n}=2 \pi f_{n}$ and $f_{n}=\frac{1}{2 \pi} \sqrt{\frac{k}{m}}$.

\subsection{Determination of $A(t)$ by $z_{\mathrm{cr}}$-based $a-v$ diagram}

In addition to the HIC, the output peak deceleration $\left(\left|\ddot{x}_{b}\right|_{\max }\right)$ (e.g. of brain tissue) and the maximum relative displacement $\left(\left|z_{b o}\right|_{\max }\right)$ (e.g. between brain tissue and head skull) can also be used to assess the occupant injury (e.g. head). In order to evaluate the influence of crash signal $A(t)$ on $\left|\ddot{x}_{b}\right|_{\text {max }}$ and $\left|z_{\text {bo }}\right|_{\text {max }}$, excitations (ESS, EPTS, ENTS, EITS, EHSS, EHVS and ENPS) shown in Fig. 2 are also used for the present discussion. Head injury is used here to illustrate the method, which may be applied to the damage/injury of other objects (occupants). Similar to the HIC-based calculation in Section 2, all crash pulses are controlled with identical $\Delta V$ and $\Delta S$ and marked with numbers in Fig.6. The deceleration is normalised by $\frac{(\Delta V)^{2}}{\Delta S}$ and time is normalised by $\frac{\Delta S}{\Delta V}$ 


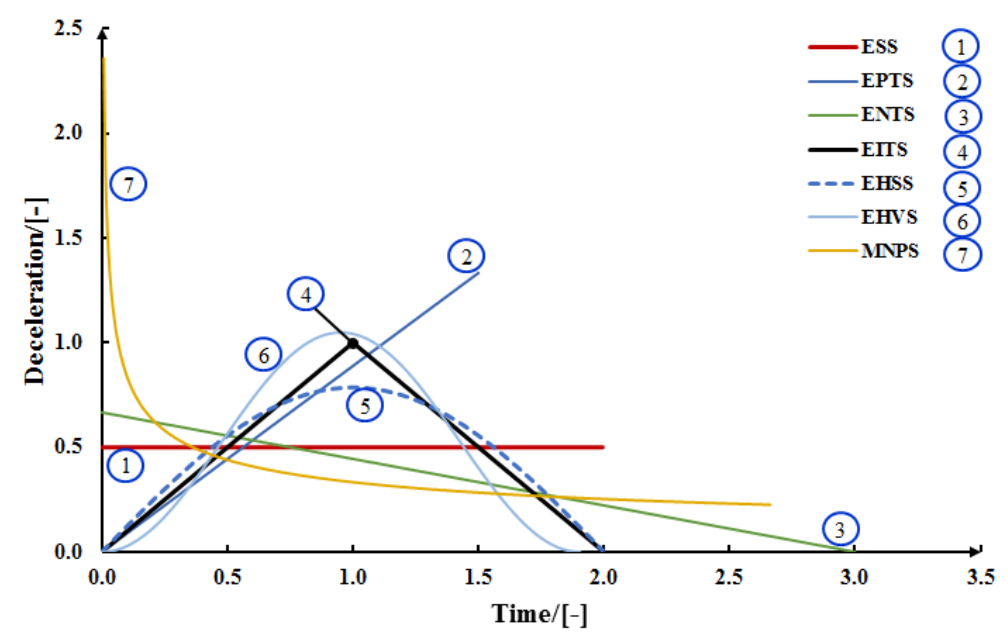

Fig.6. Normalised crash signals with identical $\Delta V$ and $\Delta S$.

SDOF model can be employed to construct the iso-damage diagram based on a critical relative displacement $\left(z_{\mathrm{cr}}\right)$. Following non-dimensional quantities are introduced to generalise the results, i.e.

$$
\left\{\begin{array}{l}
a=\frac{m A_{\max }}{z_{c r} k} \\
a_{a v g}=\frac{m A_{\text {avg }}}{z_{c r} k} \\
v=\frac{\Delta V}{z_{c r} \sqrt{k / m}}
\end{array}\right.
$$

The procedure to obtain $z_{\mathrm{cr}}$-based $a-v$ diagram is the same as that to derive P-I diagram for blast loads [23] when the pressure-time history is replaced by $A(\mathrm{t})$. The same way can be used to construct $a_{a v g}-v$ diagrams for all seven ECSs including ENPS with infinite maximum accelerations.

Fig. 7 shows the normalised $a-v$ diagrams derived from six ECSs in Fig.2 where the iso-injury diagram is determined by $\frac{z_{b o}}{z_{c r}}=1$. Fig. 8 shows the $a_{a v g}-v$ iso-injury diagram for seven ECSs. The $a-v$ diagram in Fig. 7 and the $a_{a v g}-v$ diagram in Fig.8 vary considerably with crash signal shapes in the impulsive regime. Both diagrams show that the safest boundary in the dynamic regime is determined by ESS and EPTS. In addition, ENPS has the lowest boundary for $a_{a v g}-v$ diagram in Regimes III, which means that occupants are most vulnerable in terms of injury defined by critical relative displacement when subjected to ENPS with the same velocity change and average acceleration. This conclusion is different from the results obtained in Sections 2. Therefore, it is important to clarify the conditions when considering the preferred deceleration-time history. In addition, the boundaries for ESS, ENTS and ENPS are stable in the quasi-static regime while those of other ECSs fluctuate initially, but eventually converge to a critical deceleration with 
226 EHSS and EHVS, which has been reported in the derivation of P-I diagram for explosive loading

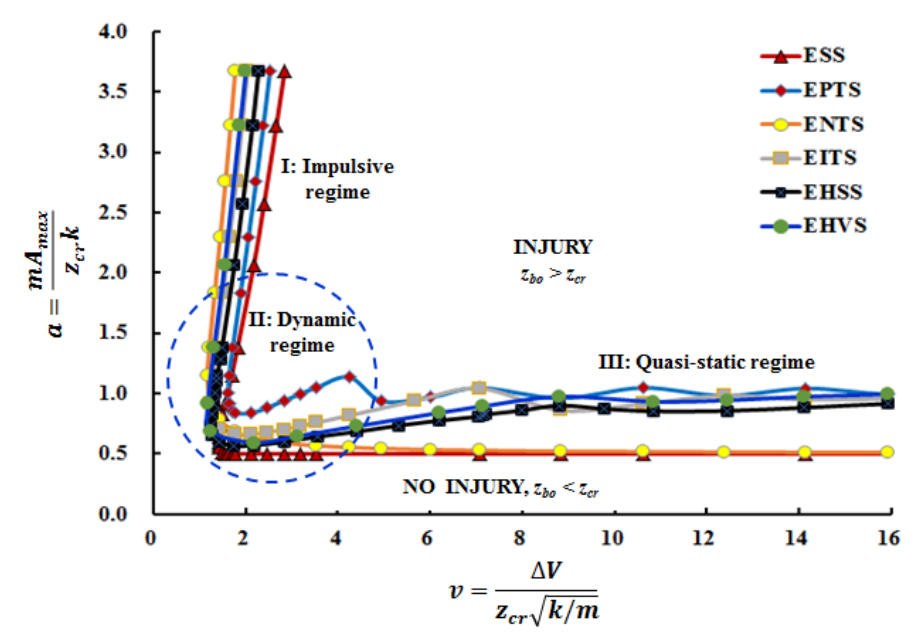

Fig.7. The normalised $a-v$ diagrams of six ECSs (ENPS is excluded).

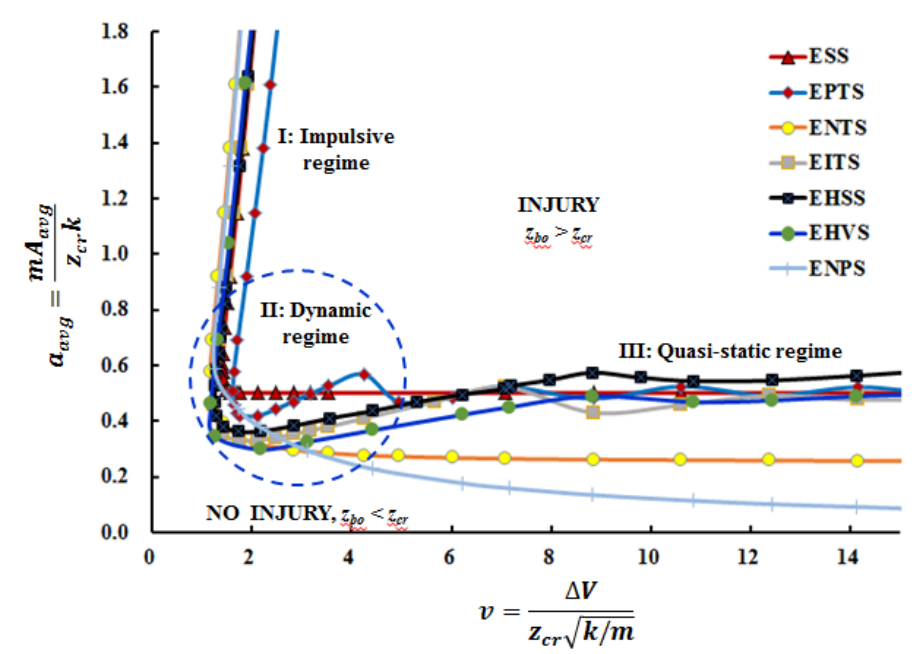

Fig.8. The normalised $a_{a v g}-v$ diagrams of seven ECSs (ENPS is included).

\subsection{Determination of $A(t)$ based on $S R S$}

Shock response spectrum (SRS) is an efficient approach to assess the severity of shock environment. Various SRS definitions have been summarised in Li and Li [30], among which, the absolute acceleration SRS (AASRS) is widely used for shock test specifications [31,32]. The AASRS is a spectrum of the maximum absolute acceleration of an array of SDOF models subjected to a base excitation. The AASRS curves derived from the seven ECSs in Fig.6 are presented to evaluate their shock severity. Here, the AASRS and structural frequency $\left(f_{n}\right)$ are normalised by $\frac{(\Delta V)^{2}}{\Delta S}$ and $\frac{\Delta V}{\Delta S}$, respectively.

As shown in Fig.9, the lowest AASRS curve is composed of the following three parts, 
(i) EPTS, $0 \leq f_{n} /\left(\frac{\Delta V}{\Delta S}\right) \leq 0.25$;

(ii) ENPS, $0.25 \leq f_{n} /\left(\frac{\Delta V}{\Delta S}\right) \leq 0.35$;

(iii) ESS, $0.35 \leq f_{n} /\left(\frac{\Delta V}{\Delta S}\right) \leq 1.00$.

244

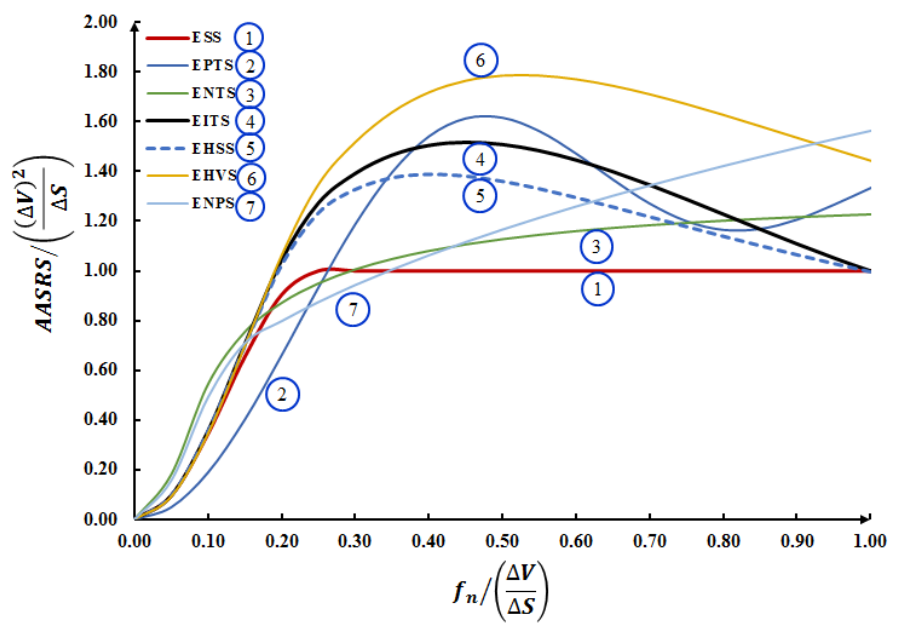

Fig.9. Normalised AASRS for various ECSs.

In addition, the relative displacement SRS (RDSRS) is also adopted for shock severity analysis, which is related to AASRS when damping is neglected [30], i.e.

$$
R D S R S=\frac{A A S R S}{\omega_{n}^{2}}
$$

As shown in Fig.10, the RDSRS normalised by $\Delta S$ exhibits the same feature in AASRS. It should be noted that SRS assessment is not related to any specified occupant or protected object.

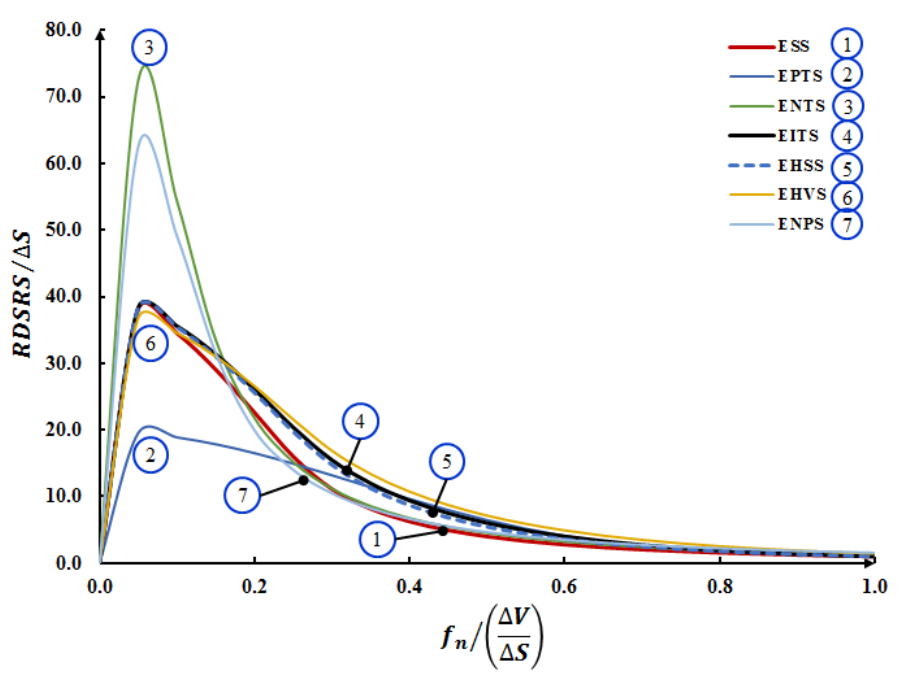

Fig.10. Normalised RDSRS plots for various ECSs. 


\section{Final Remarks}

This study made further assessment for deceleration-time histories in a crash stop from two aspects, i.e., HIC-based calculation and SDOF-based analysis. Seven well-recognised crash signals with identical velocity change $(\Delta V)$ and stop distance $(\Delta S)$ are discussed. Strong relationship between crash signal shape and occupant injury is demonstrated. Iso-injury/damage diagrams based on both HIC calculation and SDOF analysis have been built using normalised quantities. It has been found that the ENPS is the safest deceleration-time history among the seven well-recognised crash signals in terms of HIC-based calculation, which results in the safest injury boundary in $\Delta S-\Delta V$ diagram. However, EPTS, ESS and EHSS jointly construct the highest injury boundary in SDOF based $a_{a v g}-v$ diagram and SRS plots, rather than from a single crash signal. In other words, the preferred crash signal is related to the ratio between structural natural frequency and the crash signal frequency and shape. HIC is an empirical formula to assess the head injury in frontal vehicle crash. However, SDOF has broad representation of structural components, which has been widely used in structural design, such as in blast resistance design and package design. Therefore, the selection of preferred deceleration-time history in a crash stop incident depends on the priority of injury/damage criterion.

\section{Acknowledgments}

Authors would like to acknowledge the support of the China Scholarship Council (CSC) and The University of Manchester for the funding of the $\mathrm{PhD}$ work of C.X. Yang. (No. 201706370205).

\section{References}

[1] Linder A, Avery M, Krafft M, Kullgren A, Svensson MY. Acceleration pulses and crash severity in low velocity rear impacts-real world data and barrier tests. In Proceedings of the 20th International Technical Conference on the Enhanced Safety of Vehicles (CD-ROM) (No. 216) (National Highway Traffic Safety Administration, Washington, DC) 2001;1-10.

[2] Grimes WD, Lee FD. The effect of crash pulse shape on occupant simulations (No. 2000-01-0460). SAE Tech Pap, 2000.

[3] Witteman WJ, Kriens RFC. The necessity of an adaptive vehicle structure to optimize deceleration pulses for different crash velocities. In Proceedings of the 7th International Technical Conference on the Enhanced Safety of Vehicles (ESV), 2001;320:1-10.

[4] Agaram V, Xu L, Wu J, Kostyniuk G, Nusholtz G. Comparison of frontal crashes in terms of average acceleration. SAE Tech Pap, 2000.

[5] Zhou Q, Thomas MP, Stibich AM. An analytical study of system variables for meeting FMVSS 201 head impact requirements. Crashworthiness, Occupant Protection and Biomechanics in Transportation Systems, ASME, 1998;230:131-139.

[6] Huang M. Vehicle crash mechanics. CRC press, 2002. 
[7] Krafft M, Kullgren A, Ydenius A, Tingvall C. The correlation between crash pulse characteristics and duration of symptoms to the neck-crash recording in real life rear impacts. In 17th International Technical Conference on the Enhanced Safety of Vehicles 2001;1-7.

[8] Varat MS, Husher SE. Crash pulse modelling for vehicle safety research. In Proceedings: International Technical Conference on the Enhanced Safety of Vehicles (National Highway Traffic Safety Administration, Washington, DC) 2003;9-17.

[9] Urbina P, Orta P, Ahuett-Garza H. Crashworthiness design based on a simplified deceleration pulse. Int J Automot Tech 2014;15(6):909-917.

[10] Teng TL, Chang PH, Liang CC, Fung DA. Application of crash pulse on the car crashworthiness design. Adv Mech Eng 2017;9(9):1-8.

[11] Xu P, Yang CX, Peng Y, Yao SG, Zhang DH, Li BH. Crash performance and multi-objective optimization of a gradual energy-absorbing structure for subway vehicles. Int J Mech Sci 2016;107:1-12.

[12] Zhang H, Peng Y, Hou L, Tian G, Li Z. A hybrid multi-objective optimization approach for energy-absorbing structures in train collisions. Inf Sci 2019;481:491-506.

[13] Galehdari SA, Khodarahmi H. Design and analysis of a graded honeycomb shock absorber for a helicopter seat during a crash condition. Int J Crashworthiness 2016;21(3):231-241.

[14] Chou CC, Nyquist GW. Analytical studies of the head injury criterion (HIC). SAE Tech Pap,1974.

[15] Lim GG, Chou CC, Patel RN, Shahab SA, Patel PJ. Estimating the Minimum Space to Meet Federal Interior Head Impact Requirement. SAE Tech Pap, 1995.

[16] Lim GG, Chou CC, Wu F, Zhao Y. Normalization of Head Impact Test Data to $24 \mathrm{~km} / \mathrm{h}$. SAE Tech Pap, 1997.

[17] $\mathrm{Wu}$ J, Beaudet B. Optimization of head impact waveform to minimize HIC. SAE Tech Pap, 2007.

[18] Hutchinson J, Kaiser MJ, Lankarani HM. The head injury criterion (HIC) functional. Appl Math Comput 1998;96(1):1-6.

[19] Henn HW. Crash tests and the head injury criterion. Teach Math Its Appl 1998;17(4):162-170.

[20] Chichester C, Bass C, Boggess B, Davis M, Sanderson E, Di Marco G. A test methodology for assessing demining personal protective equipment (PPE) (No. RM-1). Army Communications-Electronics Command, Fort Belvoir, VA, 22060-5806, 2001.

[21] Eppinger R, Sun E, Bandak F, Haffner M, Khaewpong N, Maltese M, Kuppa S, Nguyen T, Takhounts E, Tannous R, Zhang A, Saul R. Development of improved injury criteria for the assessment of advanced automotive restraint systems-II. National Highway Traffic Safety Administration, 1999;1-70.

[22] Li QM, Meng H. Pulse loading shape effects on pressure-impulse diagram of an elastic-plastic, single-degree-of-freedom structural model. Int J Mech Sci 2002;44(9):1985-1998.

[23] Li QM, Meng H. Pressure-impulse diagram for blast loads based on dimensional analysis and single-degree-of-freedom model. J Eng Mech 2002;128(1):87-92.

[24] Krauthammer T, Astarlioglu S, Blasko J, Soh TB, Ng PH. Pressure-impulse diagrams for the behavior assessment of structural components. Int J Impact Eng 2008;35(8):771-783.

[25] Abedini M, Mutalib AA, Raman SN, Alipour R, Akhlaghi E. Pressure-Impulse (P-I) Diagrams for Reinforced Concrete (RC) Structures: A Review. Arch Comput Methods Eng 2018:1-35.

[26] Kipp WI. Developments in testing products for distribution. Packaging Technology and Science. 2000 May;13(3):89-98.

[27] Goyal S, Papadopoulos JM, Sullivan PA. Shock protection of portable electronic products: shock response spectrum, damage boundary approach, and beyond. Shock and Vibration. 1997 Jan 1;4(3):169-91. 
[28] Qian J, Cai H, Ma W, Hao Z. Shock analysis on a packaged washing machine from damage boundary: shock response spectrum to component failure. Shock and Vibration. 2015;2015.

[29] American Society for Testing and Materials, "ASTMD3332, standard test methods for mechanical shock fragility of products, using shock machines," in Annual Book of ASTM Standards, 2010.

[30] Li BW, Li QM. Damage boundary of structural components under shock environment. Int J Impact Eng 2018;118:67-77.

[31] NASA-STD-7003A. Pyroshock test criteria. NASA Technical Standard. National Aeronautics and Space Administration, USA, 2011.

[32] MIL-STD-810 G, "Department of Defense, Test Method Standard, Environmental Engineering Considerations and Laboratory Tests", Method 522.1, pages 522.1-1 through 522.1-15. 